\title{
On computing pi-coefficients
}

\author{
Jinn-Fwu Wu, Jia-Sheng Heh, \\ and I-Kong Fong
}

Department of Electrical Engineering, National Taiwan University, Taipei, Taiwan 10764, ROC

Received 6 July 1990

Revised 24 November 1990

Abstract: Introduced by Lawrence and Johnson in 1986, the concept of pi-sharing can be used to analyze the stability of discrete-time systems. However, to determine the suitable picoefficients required in the application of pi-sharing theory is in general a difficult task. It involves checking the negative semi-definiteness (n.s.d.) of a complicated matrix. This paper formulates an alternative problem, the max-p problem, for determining the 'best' pi-coefficients, and proposes a solution method for the problem. The n.s.d. condition is turned into conditions on the coefficients of a series of polynomials, which are easier to solve than the original one. An example is used to illustrate the procedure in detail.

Keywords: Pi-sharing theory; pi-coefficients: passivity; hyperstability; strictly positive realness.

\section{Introduction}

Generalizing the concepts of passivity and hyperstability [2], Lawrence and Johnson develop the pi-sharing theory to analyze the stability of discrete-time systems, including the recursive identification process and adaptive control systems $[1,3,4]$. The theory uses a set of pi-coefficients to describe the system characteristics based on the concept of energy exchange. With the theory, it can be shown that a feedback system consisting of two subsystems in a loop, even though one of the subsystems is not passive, can still be stable by the dissipativeness of the other subsystem. When applied to the error model analyses $[5-7]$ of adaptive systems, this theory can deduce quite meaningful stability conditions [3]. The SPR (strictly positive realness) condition for the linear time-invariant subsystem, and the passivity condition for the nonlinear time-varying subsystem obtained by the traditional passivity theorem, hyperstability theory, and the Lyapunov method, are all special cases of pi-sharing stability conditions $[1,3]$. Moreover, the SPR condition can be relaxed by the 'persistent power' condition [3].

Although through the quantitative descriptions of pi-coefficients, the pi-sharing theory does have much potential to provide more definite interpretations of an adaptive control system, recent research about this topic is rare, partly because it is hard to determine suitable pi-coefficients, and with an inappropriate choice the resulting stability analysis may be too conservative or even useless $[1,3]$. In this paper, aiming at the purpose of maximizing the finite mean-square gain stability range of the type of feedback systems mentioned above, we propose an alternative formulation for obtaining a set of pi-coefficients, which is called the max-p problem. In the original work [1], the determination of suitable pi-coefficients involves checking the n.s.d. of a complicated matrix, while in our solution method for the max-p problem, it becomes checking some conditions, mainly the Hurwitzness, on a series of polynomials. It is clear, at least from the example we use to illustrate the procedure, that our formulation and solution method do provide an easier way for finding the pi-coefficients. Furthermore, from the very purpose of forming the max-p problem, the set of pi-coefficients obtained in this paper is seen to have a certain optimal sense.

\section{Problem formulation}

Consider a single-input-single-output discretetime system with a state-space-like 'factorable' form [3]:

$$
\begin{aligned}
& \boldsymbol{x}(k+1)=\boldsymbol{A}(k) \boldsymbol{x}(k)+\boldsymbol{b}(k) u(k), \\
& y(k)=\boldsymbol{c}^{\mathrm{T}}(k) \boldsymbol{x}(k)+d(k) u(k),
\end{aligned}
$$

where $\boldsymbol{x}(k) \in \mathbb{R}^{n}$ is the state vector, $u(k) \in \mathbb{R}$ is the input, $y(k) \in \mathbb{R}$ is the output, and $\boldsymbol{A}(k)$, $\boldsymbol{b}(k), \boldsymbol{c}(k)$ and $d(k)$ are system 'matrices' with suitable dimensions. The system (1), (2) is said to 
be pi-sharing with respect to pi-coefficients $\{\Gamma(k), Q(k), p(k), r(k)\}[1]$ if

$$
\begin{aligned}
& \sum_{k=0}^{\tau} u(k) y(k) \\
& \geq \boldsymbol{x}^{\mathrm{T}}(\tau+1) \Gamma(\tau+1) \boldsymbol{x}(\tau+1) \\
& \quad-\boldsymbol{x}^{\mathrm{T}}(0) \Gamma(0) \boldsymbol{x}(0)+\sum_{k=0}^{\tau} \boldsymbol{x}^{\mathrm{T}}(k) \boldsymbol{Q}(k) \boldsymbol{x}(k) \\
& \quad+\sum_{k=0}^{\tau} y^{2}(k) p(k)+\sum_{k=0}^{\tau} u^{2}(k) r(k)
\end{aligned}
$$

for all $\tau \in \mathbb{N}$ and ( $u, x, y$ ) satisfying (1), (2), and both $\Gamma(k)$ and $Q(k)$ are positive semi-definite (p.s.d) for $0 \leq k \leq \tau+1$. A sufficient condition [1] for $\{\Gamma(\cdot), Q(\cdot), p(\cdot), r(\cdot)\}$ to be a set of pi-coefficients for the system (1), (2) is that

$\boldsymbol{M}(k)=\left[\begin{array}{ll}\boldsymbol{M}_{1}(k) & \boldsymbol{m}_{2}(k) \\ \boldsymbol{m}_{2}^{\mathrm{T}}(k) & m_{4}(k)\end{array}\right]$,

is n.s.d. for $0 \leq k \leq \tau$, where

$$
\begin{aligned}
\boldsymbol{M}_{1}(k)= & \boldsymbol{A}^{\mathrm{T}}(k) \Gamma(k+1) \boldsymbol{A}(k)-\Gamma(k) \\
& +\boldsymbol{Q}(k)+p(k) \boldsymbol{c}(k) \boldsymbol{c}^{\mathrm{T}}(k), \\
\boldsymbol{m}_{2}(k)= & \boldsymbol{A}^{\mathrm{T}}(k) \Gamma(k+1) \boldsymbol{B}(k) \\
& -\frac{1}{2} \boldsymbol{c}(k)+p(k) d(k) \boldsymbol{c}(k), \\
m_{4}(k)= & \boldsymbol{b}^{\mathrm{T}}(k) \Gamma(k+1) \boldsymbol{b}(k) \\
& -d(k)+r(k)+p(k) d^{2}(k) .
\end{aligned}
$$

Consider a composite system $\subseteq$ as shown in Figure 1. Suppose the forward-path subsystem $\Xi_{1}$ has pi-coefficients $\left\{\Gamma_{1}(k), Q_{1}(k), p_{1}(k), r_{1}(k)\right\}$ and the feedback-path subsystem $\subseteq_{2}$ has pi-coefficients $\left\{\Gamma_{2}(k), \boldsymbol{Q}_{2}(k), p_{2}(k), r_{2}(k)\right\}$. Then a set of sufficient conditions ensuring $\subseteq$ has finite mean-square gain [1] is

(a) $r_{1}(k)+p_{2}(k)>0, \quad \forall k \in \mathbb{N}$,

and

(b) $p_{1}(k)+r_{2}(k)>0, \quad \forall k \in \mathbb{N}$.

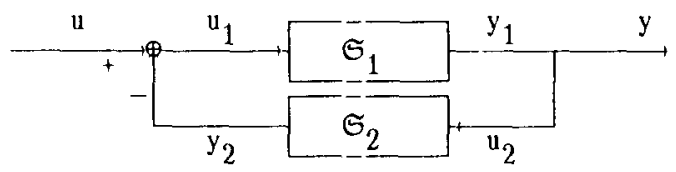

Fig. 1. Composite system.
On the other hand, this means that as long as the energy generation $(p<0$ or $r<0)$ in one subsystem is overcome by the excess dissipation $(p>0$ or $r>0$ ) in the other, the interconnected system remains stable. On the other hand, this also means that one subsystem having too small (or even negative) pi-coefficients will impose stringent stability conditions upon the other. Therefore, to maximize the finite mean-square gain stability range for the system $\cong$, it is advisory to find the sets of pi-coefficients for $\Xi_{1}$ and $\Xi_{2}$ with $p_{i}$ 's and $r_{i}$ 's as large as possible. However, the pi-coefficients $p$ and $r$ for a subsystem are themselves correlated as can be seen from the expression for the matrix $\boldsymbol{M}$, which is required to be n.s.d. Hence, a practical way for finding the suitable pi-coefficients is to maximize $p$ (or $r$ ) with $r$ (or $p$ ) required to be greater than or equal to a preset constant. This brings out our formulation of finding for a system the 'optimal' pi-coefficients. For simplicity, we shall neglect the time index $k$ in the following discussion, i.e., we shall consider only time-invariant pi-coefficients. Our information is:

Problem 1 (Max-p problem).

$\underset{\Gamma, Q, r}{\operatorname{Maximize} p}$

subject to
(a) $\boldsymbol{M} \leq \mathbf{0}$,
(b) $\Gamma \geq \mathbf{0}, \boldsymbol{Q} \geq \mathbf{0}$, and $r \geq r_{0}$, a given constant.

\section{Properties of $M$-matrix}

Clearly, in the max-p problem, condition (a) is more difficult to satisfy than condition (b). Hence we shall analyze the properties of the matrix $\boldsymbol{M}$, trying to change condition (a) into a more solvable condition. We first regroup $\boldsymbol{M}$ in (4)-(7) as

$\boldsymbol{M}=\boldsymbol{E}+p \cdot \boldsymbol{H}$,

where

$\boldsymbol{E}=\left[\begin{array}{cc}\boldsymbol{A}^{\mathrm{T}} \Gamma \boldsymbol{A}-\Gamma+\boldsymbol{Q} & \boldsymbol{A}^{\mathrm{T}} \Gamma \boldsymbol{b}-\frac{1}{2} \boldsymbol{c} \\ \boldsymbol{b}^{\mathrm{T}} \Gamma \boldsymbol{A}-\frac{1}{2} \boldsymbol{c}^{\mathrm{T}} & \boldsymbol{b}^{\mathrm{T}} \Gamma \boldsymbol{b}-d+r\end{array}\right]$,

and

$\boldsymbol{H}=\left[\begin{array}{l}\boldsymbol{c} \\ d\end{array}\right] \cdot\left[\begin{array}{ll}\boldsymbol{c}^{\mathrm{T}} & d\end{array}\right] \equiv \boldsymbol{h} \cdot \boldsymbol{h}^{\mathrm{T}}$. 
It is obvious that $\boldsymbol{M}$ is affine in $p$, and $\boldsymbol{M}, \boldsymbol{E}$, and $\boldsymbol{H}$ are all symmetric matrices. We derive the following result first.

Lemma 1. For the matrices defined in (13)-(15),

(a) $\operatorname{det}(\lambda \cdot \boldsymbol{I}-\boldsymbol{M})=f(\lambda)-p \cdot g(\lambda)$, where $f(\lambda)=\operatorname{det}(\lambda I-E)$ and $g(\lambda)=\boldsymbol{h}^{\mathrm{T}} \operatorname{adj}(\lambda \boldsymbol{I}-\boldsymbol{E})$. $\boldsymbol{h}$; and

(b) $g(\lambda)=\left(\boldsymbol{h}^{\mathrm{T}} \boldsymbol{h}\right) \operatorname{det}\left(\lambda \boldsymbol{I}-\tilde{\boldsymbol{E}}_{1}\right)$, where $\tilde{\boldsymbol{E}}_{1}$ is the leading $(n-1) \times(n-1)$ principal submatrix of $\tilde{\boldsymbol{E}}=\boldsymbol{U E} \boldsymbol{U}^{\mathrm{T}}$, and $\boldsymbol{U}$ is an orthogonal matrix.

Proof. (a) By the definitions,

$$
\begin{aligned}
& \operatorname{det}(\lambda \boldsymbol{I}-\boldsymbol{M}) \\
& =\operatorname{det}(\lambda \boldsymbol{I}-\boldsymbol{E}-p \boldsymbol{H}) \\
& =\operatorname{det}(\lambda \boldsymbol{I}-\boldsymbol{E}) \cdot \operatorname{det}\left[\boldsymbol{I}-p(\lambda \boldsymbol{I}-\boldsymbol{E})^{-1} \boldsymbol{h} \boldsymbol{h}^{\mathrm{T}}\right] \\
& =\operatorname{det}(\lambda \boldsymbol{I}-\boldsymbol{E}) \cdot\left[1-p \boldsymbol{h}^{\mathrm{T}}(\lambda \boldsymbol{I}-\boldsymbol{E})^{-1} \boldsymbol{h}\right] \\
& =\operatorname{det}(\lambda \boldsymbol{I}-\boldsymbol{E})-p \boldsymbol{h}^{\mathrm{T}} \operatorname{adj}(\lambda \boldsymbol{I}-\boldsymbol{E}) \cdot \boldsymbol{h} .
\end{aligned}
$$

(b) From the expression for $g(\lambda)$ in (a), it is easy to see that

$g(\lambda)=\operatorname{det}\left[\begin{array}{cc}\lambda \boldsymbol{I}-\boldsymbol{E} & -\boldsymbol{h} \\ \boldsymbol{h}^{\mathrm{T}} & 0\end{array}\right]$.

There exists an orthogonal matrix $U$ (obtainable by pre-multiplying a suitable Householder transformation [8] with the backward identity matrix, i.e., the matrix with unity elements on its anti-diagonal and zero elements elsewhere) such that $\boldsymbol{U} \cdot \boldsymbol{h}=\sqrt{\boldsymbol{h}^{\mathrm{T}} \boldsymbol{h}} \cdot \boldsymbol{e}_{n+1}$, where $\boldsymbol{e}_{n+1}$ is the last column of the $(n+1) \times(n+1)$ identity matrix. Thus

$$
\begin{aligned}
g(\lambda) & =\operatorname{det}\left(\left[\begin{array}{cc}
\lambda \boldsymbol{I}-\boldsymbol{U} \boldsymbol{E} \boldsymbol{U}^{\mathrm{T}} & -\boldsymbol{U} \boldsymbol{h} \\
\boldsymbol{h}^{\mathrm{T}} \boldsymbol{U}^{\mathrm{T}} & 0
\end{array}\right]\right) \\
& =\left(\boldsymbol{h}^{\mathrm{T}} \boldsymbol{h}\right) \operatorname{det}\left(\left[\begin{array}{cc}
\lambda \boldsymbol{I}-\tilde{\boldsymbol{E}} & -\boldsymbol{e}_{n+1} \\
\boldsymbol{e}_{n+1}^{\mathrm{T}} & 0
\end{array}\right]\right) \\
& =\left(\boldsymbol{h}^{\mathrm{T}} \boldsymbol{h}\right) \operatorname{det}\left(\lambda \boldsymbol{I}-\tilde{\boldsymbol{E}}_{1}\right) .
\end{aligned}
$$

From the lemma above, it is known that the degrees of $f(\lambda)$ and $g(\lambda)$ and $n+1$ and $n$ (consider the non-trivial case in which $\boldsymbol{h} \neq \mathbf{0}$ ), respectively. Let $f(\lambda)$ and $g(\lambda)$, respectively, be decomposed as

$f(\lambda)=\prod_{i=1}^{n+1}\left(\lambda-\alpha_{i}\right)$, and

$$
g(\lambda)=\left(\boldsymbol{h}^{\mathrm{T}} \boldsymbol{h}\right) \prod_{i=1}^{n}\left(\lambda-\beta_{i}\right)
$$

Define

$\psi(\lambda)=f(\lambda) / g(\lambda)$

and

$$
\begin{aligned}
\mu(\lambda) & =\operatorname{det}(\lambda \boldsymbol{I}-\boldsymbol{M})=f(\lambda)-p \cdot g(\lambda) \\
& =\prod_{i=1}^{n+1}\left(\lambda-\gamma_{i}\right) .
\end{aligned}
$$

Then we have:

Lemma 2. All the roots of $f(\lambda), g(\lambda)$, and $\mu(\lambda)$ are real, i.e., $\alpha_{i} \in \mathbb{R}$ for $i=1, \ldots, n+1, \beta_{i} \in \mathbb{R}$ for $i=1, \ldots, n$, and $\gamma_{i} \in \mathbb{R}$ for $i=1, \ldots, n+1$.

Proof. The realness of the $\alpha_{i}$ 's, $\beta_{i}$ 's, and $\gamma_{i}$ 's can be obtained easily from the fact that the matrices $\boldsymbol{E}, \tilde{\boldsymbol{E}}, \tilde{\boldsymbol{E}}_{1}$, and $\boldsymbol{M}$ are all symmetric.

Without loss of generality, we let

$\alpha_{1} \leq \alpha_{2} \leq \cdots \leq \alpha_{n} \leq \alpha_{n+1}$,

$\beta_{1} \leq \beta_{2} \leq \cdots \leq \beta_{n}$,

and

$\gamma_{1} \leq \gamma_{2} \leq \cdots \leq \gamma_{n} \leq \gamma_{n+1}$.

To obtain our next result, Theorem 1 , we need the following lemma [8].

Lemma 3. If $\boldsymbol{A}_{i}$ denotes the leading $i \times i$ principal submatrix of an $n \times n$ symmetric matrix $A$, then for $i=1,2, \ldots, n-1$, the following interlacing property holds:

$$
\begin{aligned}
\lambda_{1}\left(\boldsymbol{A}_{i+1}\right) & \leq \lambda_{1}\left(\boldsymbol{A}_{i}\right) \leq \cdots \leq \lambda_{i}\left(\boldsymbol{A}_{i+1}\right) \\
& \leq \lambda_{i}\left(\boldsymbol{A}_{i}\right) \leq \lambda_{i+1}\left(\boldsymbol{A}_{i+1}\right),
\end{aligned}
$$

where $\lambda_{j}(\boldsymbol{A})$ denotes the $j$-th smallest eigenvalue of $\boldsymbol{A}$.

Theorem 1. (a) The roots of $f(\lambda)$ and $g(\lambda)$ interlace, i.e.,

$$
\begin{aligned}
\alpha_{1} & \leq \beta_{1} \leq \alpha_{2} \leq \cdots \leq \alpha_{i} \leq \beta_{i} \\
& \leq \alpha_{i+1} \leq \cdots \leq \alpha_{n} \leq \beta_{n} \leq \alpha_{n+1}
\end{aligned}
$$


(b) $\psi(\lambda)$ is continuous in $\left(\beta_{i-1}, \beta_{i}\right)$ where $\beta_{i-1}$ $<\beta_{i}$. For the sake of consistency we let $\beta_{0} \equiv-\infty$ and $\beta_{n+1} \equiv+\infty$.

(c) $\psi(\lambda)>0$ for $\lambda \in\left(\alpha_{i}, \beta_{i}\right)$ where $\alpha_{i}<\beta_{i}$; whereas $\psi(\lambda)<0$ for $\lambda \in\left(\beta_{i-1}, \alpha_{i}\right)$ where $\beta_{i-1}<$ $\alpha$.

(d) $\psi(\lambda)$ is monotonically increasing in $\left(\beta_{i-1}, \beta_{i}\right)$ where $\beta_{i-1}<\beta_{i}$.

(e) For all $p>0, \gamma_{i} \in\left[\alpha_{i}, \beta_{i}\right]$; whereas for all $p<0, \gamma_{i} \in\left[\beta_{i-1}, \alpha_{i}\right]$.

Proof. (a) From Lemma 3, the roots of $g(\lambda)=\left(\boldsymbol{h}^{\mathrm{T}} \boldsymbol{h}\right) \operatorname{det}\left(\lambda \boldsymbol{I}-\tilde{\boldsymbol{E}}_{1}\right)$

have the interlacing properties with the eigenvalues of $\tilde{E}$, which are the same as those of $E$, i.e., the roots of $f(\lambda)=\operatorname{det}(\lambda \boldsymbol{I}-\boldsymbol{E})$.

(b) Since $\psi(\lambda)$ is a rational function, it is continuous in the intervals where its denominator is not zero. This proves the statement in (b). Note that it may happen that $\beta_{i}=\beta_{i+1}$ for some $i$. In general, suppose

$\beta_{i-1}<\beta_{i}=\beta_{i+1}=\cdots=\beta_{i+j}<\beta_{i+j+1}$;

then we must have

$\beta_{i}=\alpha_{i+1}=\beta_{i+1}=\cdots=\alpha_{i+j}=\beta_{i+j}$

by the interlacing property (a). Hence there must be at least $j$ 'pole-zero cancellations' in $\psi(\lambda)$. If $\alpha_{i}$ or $\alpha_{i+j+1}$ equals $\beta_{i}$, then $\psi(\lambda)$ is not continuous in the intervals $\left(\beta_{i-1}, \beta_{i}\right)$ and $\left(\beta_{i+j}, \beta_{i+j+1}\right)$, i.e., $\left(\beta_{i}, \beta_{i+j+1}\right)$, but also continuous at the point $\lambda=\beta_{i}$.

(c) Note that both $f(\lambda)$ and $g(\lambda)$ have positive leading coefficients (unity and $\boldsymbol{h}^{\mathrm{T}} \boldsymbol{h}$, respectively). In addition, for $\lambda \in\left(\alpha_{i}, \beta_{i}\right)$ where $\alpha_{i}<\beta_{i}$, both

$$
\frac{\left(\lambda-\alpha_{1}\right) \cdots\left(\lambda-\alpha_{i}\right)}{\left(\lambda-\beta_{1}\right) \cdots\left(\lambda-\beta_{i-1}\right)}
$$

and

$$
\frac{\left(\lambda-\alpha_{i+1}\right) \cdots\left(\lambda-\alpha_{n+1}\right)}{\left(\lambda-\beta_{i}\right) \cdots\left(\lambda-\beta_{n}\right)}
$$

are positive. Thus $\psi(\lambda)$ is positive in $\left(\alpha_{i}, \beta_{i}\right)$. The second part of (c) can be proved similarly.

(d) Let

$$
\begin{aligned}
\frac{\mathrm{d} \psi(\lambda)}{\mathrm{d} \lambda} & =\psi(\lambda)\left(\sum_{j=1}^{n+1} \frac{1}{\lambda-\alpha_{j}}-\sum_{j=1}^{n} \frac{1}{\lambda-\beta_{j}}\right) \\
& \equiv \psi(\lambda) \Delta(\lambda) .
\end{aligned}
$$

Since for $\lambda \in\left(\beta_{j-1}, \alpha_{i}\right)$,

$\Delta(\lambda)=\sum_{j=1}^{n}\left(\frac{1}{\lambda-\alpha_{j}}-\frac{1}{\lambda-\beta_{j}}\right)+\frac{1}{\lambda-\alpha_{n+1}}<0$,

and for $\lambda \in\left(\alpha_{i}, \beta_{i}\right)$,

$\Delta(\lambda)=\frac{1}{\lambda-\alpha_{1}}+\sum_{j=1}^{n}\left(\frac{1}{\lambda-\alpha_{j+1}}-\frac{1}{\lambda-\beta_{j}}\right)>0$,

we have for $\lambda \in\left(\beta_{i-1}, \alpha_{i}\right) \cup\left(\alpha_{i}, \beta_{i}\right)$ that $\mathrm{d} \psi(\lambda) / \mathrm{d} \lambda>0$. The point $\lambda=\alpha_{i}$, if $\beta_{i-1}<\alpha_{i}<\beta_{i}$, can be discussed similarly. We omit it for the sake of brevity.

(e) Consider first the simplest case in which $\beta_{i-1}<\alpha_{i}<\beta_{i}$ for $i=1, \ldots, n+1$. Since $\mu(\lambda)=$ $f\left(\lambda-p \cdot g(\lambda)\right.$, it is easy to see that $\gamma_{i} \notin$ $\left\{\beta_{1}, \ldots, \beta_{n}\right\}$ for $i=1, \ldots, n+1$. Thus

$\mu(\lambda)=g(\lambda)[\psi(\lambda)-p]=0$

if and only if $\psi(\lambda)=p$. Utilizing the properties of $\psi(\lambda)$ described in (a)-(d), we can conclude that the values of $\lambda$ at which $\psi(\lambda)=p$ satisfy (e). In general, if

$\beta_{i-1}<\beta_{i}=\cdots=\beta_{i+j}<\beta_{i+j+1}$,

then by the interlacing property (a) and the fact that $\mu(\lambda)=f(\lambda)-p \cdot g(\lambda)$, we have at least $\gamma_{t}=$ $\alpha_{t}=\beta_{i}$ for $t=i+1, \ldots, i+j$. After these 'common roots' are extracted, the remaining discussion is the same as that for the simplest case.

To make some of the statements made in the proof of Theorem 1 and some subsequent statements more clear, Figure 2 displays the $\psi(\lambda)$ for

$f(\lambda)=(\lambda+6)(\lambda+4)(\lambda+3)(\lambda+1)$

and

$g(\lambda)=(\lambda+5)(\lambda+3)(\lambda+2)$

as an example. It is seen that the diagram of $\psi(\lambda)$ consists of several 'branches', each being confined between two adjacent uncancelled poles (roots of $g$ ), and monotonically increasing from $-\infty$ to $+\infty$. Also, with every horizontal (dotted) line corresponding to a constant value of $p$, each branch has exactly one intersection.

Continuing our discussion on the n.s.d. condi- 
tions of the matrices $\boldsymbol{M}$, we let $\nu_{g}$ and $\nu_{f}$ denote, respectively, the number of zero roots of $g(\lambda)$ and $f(\lambda)$. Then

$$
g(\lambda)=\lambda^{v_{s}}\left(g_{n} \lambda^{n-v_{k}}+\cdots+g_{v_{g}}\right) \equiv \lambda^{\nu_{*}} \tilde{g}(\lambda),
$$

and

$$
\begin{aligned}
f(\lambda) & =\lambda^{\nu_{f}}\left(\lambda^{n-v_{f}+1}+f_{n} \lambda^{n-v_{f}}+\cdots+f_{\nu_{f}}\right) \\
& \equiv \lambda^{\nu_{f}} \tilde{f(\lambda),}
\end{aligned}
$$

where $g_{v_{g}} \neq 0$ and $f_{\nu_{f}} \neq 0$. From the interlacing property of Theorem 1, we know

$\nu_{g}-1 \leq \nu_{j} \leq \nu_{g}+1$.

Moreover, we have:

Theorem 2. There exists a finite $p$ such that the matrix $M$ is n.s.d. if and only if $\tilde{g}(\lambda)$ is strictly Hurwitz and $\nu_{g} \leq \nu_{g}$.

Proof. Note first that the matrix $\boldsymbol{M}$ is n.s.d. if and only if its eigenvalues $\gamma_{i} \leq 0$ for all $i=1, \ldots, n+1$. Also, in the proof of Theorem 1 we see that, for a given $p$, the eigenvalues of $\boldsymbol{M}$ are either the common roots of $f(\lambda)$ and $g(\lambda)$, or the value at which $\psi(\lambda)=p$.

To show the 'if' part, we see that if $\tilde{g}(\lambda)$ is strictly Hurwitz, then the largest common roots of $f(\lambda)$ and $g(\lambda)$ are non-positive. In addition, except the rightmost branch of $\psi(\lambda)$, all others are confined to the left of $\lambda=0$. Now, if $\nu_{g} \leq \nu_{f}$, then the largest uncancelled pole of $\psi(\lambda)$ is negative. Hence the rightmost branch of $\psi(\lambda)$ has a part to the left of $\lambda=0$, and $\psi(0)$ is equal to the (finite) maximal $p$ that makes the matrix $\boldsymbol{M}$ n.s.d.

To show the 'only if' part, we see that if $\tilde{g}(\lambda)$ is not Hurwitz, then either $g(\lambda)$ and $f(\lambda)$ have some common positive roots, or there is at least one branch of $\psi(\lambda)$ completely located to the right of $\lambda=0$, both resulting in positive eigenvalues of $\boldsymbol{M}$ for any finite $p$. Suppose now $\tilde{g}(\lambda)$ is Hurwitz and $\nu_{g}-1=\nu_{f}$. Then there remains an uncanceled pole of $\psi(\lambda)$ at $\lambda=0$. This implies the rightmost branch of $\psi(\lambda)$ is completely to the right of $\lambda=0$. Again we have a positive eigenvalue of $\boldsymbol{M}$ for any finite $p$.

\section{Solution of the max-p problem}

From the proof of Theorem 2, we immediately obtain:

Corollary 1. The max-p problem (10)-(12) can be reformulated as:

\section{Problem 2.}

$$
\underset{\Gamma, \boldsymbol{Q}, r}{\operatorname{Maximize}} \frac{f_{v_{g}}}{g_{v_{g}}}
$$

subject to

(a) $\tilde{g}(\lambda)$ is Hurwitz and $\nu_{g} \leq \nu_{f}$,

(b) $\Gamma \geq \mathbf{0}, \boldsymbol{Q} \geq \mathbf{0}$, and $r \geq r_{0}$, a given constant.

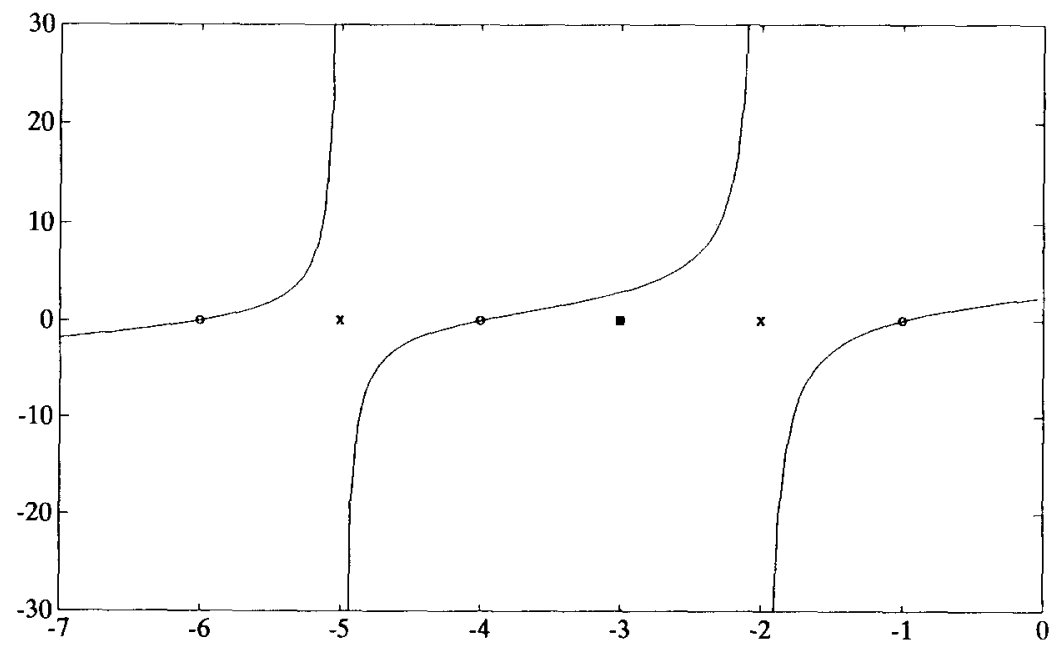

Fig. 2. Branches of $\psi(\lambda)$. 
Note that we use $f_{v_{g}}$ to denote the coefficient of

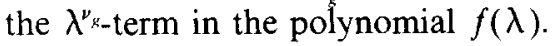

Proof. By Theorem 2, the condition (11) in the max-p problem can be replaced by the conditions in (28). Also, if $v_{g}=v_{f}$, then

$\psi(0)=f_{v_{f}} / g_{v_{k}}=f_{v_{g}} / g_{v_{g}}$

is the maximal value of $p$ for the n.s.d. of $\boldsymbol{M}$. Otherwise $\nu_{g}+1=v_{f}$, which implies $f_{\nu_{p}}=0$ and thus $\psi(0)=0$ is the maximal value of $p$ for the n.s.d. of $M$. In both these two cases the new objective function $f_{v_{x}} / g_{v_{x}}$ matches the original objective function $p$.

Let

$\Omega_{i} \equiv\left\{(\Gamma, \boldsymbol{Q}, r) \mid \Gamma \geq \mathbf{0}, \boldsymbol{Q} \geq \mathbf{0}, r \geq r_{0}\right.$,

$\tilde{g}(\lambda)$ is strictly Hurwitz, and $\left.i=\nu_{g} \leq \nu_{f}\right\}$

for $0 \leq i \leq n$. Obviously the feasible region, constrained by (28) and (29), of Problem 2 is equal to $\Omega_{0} \cup \cdots \cup \Omega_{n}$. Thus we may sequentially maximize the objective function in (27) subject to the constraint that $(\Gamma, Q, r) \in \Omega_{i}$, for $i=0, \ldots, n$. Note that because of the strict Hurwitzness condition on $\tilde{g}(\lambda), \Omega_{i}$ is in general not a closed set. Therefore the objective function (27) may have a supremum rather than a maximum in $\Omega_{i}$. However, our numerical experiences show that if we find a supremum, rather than a maximum, in $\Omega_{i}$, then the supremum can always be obtained as a local maximum in $\Omega_{i+1}$. Thus currently we only compare all the resultant maximal values from maximizations over $\Omega_{i}$ 's with one another. The set of $(\Gamma, Q, r)$ corresponding to the largest maximum, together with the maximum, form the 'optimal' set of pi-coefficients.

An example is used to illustrate this process.

Example 1. Consider the first-order system

$\zeta\left(z^{-1}\right)=\kappa \frac{1+b_{1} z^{-1}}{1-a_{1} z^{-1}}$,

whose system 'matrices' are $\boldsymbol{A}=a_{1}, \boldsymbol{b}=\kappa, \boldsymbol{c}=a_{1}$ $+b_{1}$, and $d=\kappa$. Then, $\boldsymbol{M}=\boldsymbol{E}+p \boldsymbol{H}$ where

$\boldsymbol{E}=\left(\begin{array}{cc}\left(a_{1}^{2}-1\right) \Gamma+\boldsymbol{Q} & \kappa a_{1} \Gamma-\frac{1}{2}\left(a_{1}+b_{1}\right) \\ \kappa a_{1} \Gamma-\frac{1}{2}\left(a_{1}+b_{1}\right) & \kappa^{2} \Gamma-\kappa+r\end{array}\right)$,

$\boldsymbol{H}=\left(\begin{array}{cc}\left(a_{1}+b_{1}\right)^{2} & \kappa\left(a_{1}+b_{1}\right) \\ \kappa\left(a_{1}+b_{1}\right) & \kappa^{2}\end{array}\right)$.
By Lemma 1,

$f(\lambda)=\lambda^{2}+f_{1} \lambda+f_{0}=\operatorname{det}(\lambda \boldsymbol{I}-\boldsymbol{E})$,

and

$g(\lambda)=g_{1} \lambda+g_{0}$.

Then,

$$
\begin{aligned}
f_{0}= & -\kappa^{2} \Gamma^{2}+\left[\kappa\left(1+a_{1} b_{1}\right)-r\left(1-a_{1}^{2}\right)+\kappa^{2} \boldsymbol{Q}\right] \Gamma \\
& -\frac{1}{4}\left(a_{1}+b_{1}\right)^{2}+(r-\kappa) \boldsymbol{Q}, \\
g_{0}= & \kappa^{2}\left(1-b_{1}^{2}\right) \Gamma-r\left(a_{1}+b_{1}\right)^{2}-\kappa^{2} \boldsymbol{Q}, \\
f_{1}= & \left(1-a_{1}^{2}-\kappa^{2}\right) \Gamma-r+\kappa-Q, \\
g_{1}= & \kappa^{2}+\left(a_{1}+b_{1}\right)^{2} .
\end{aligned}
$$

To solve Problem 2 in the feasible region $\Omega_{0}$ is to

$\max f_{0} / g_{0}$

subject to $\Gamma \geq \mathbf{0}, \boldsymbol{Q} \geq \mathbf{0}, r \geq r_{0}, g_{0}>0$.

We take the partial derivatives of the objective function with respect to each independent variable. Then

$$
\begin{aligned}
\frac{\partial\left(f_{0} / g_{0}\right)}{\partial \boldsymbol{Q}} & =-\frac{\left[\kappa^{2} b_{1} \Gamma+\left(r-\frac{1}{2} \kappa\right)\left(a_{1}+b_{1}\right)\right]^{2}}{g_{0}^{2}} \\
& <0
\end{aligned}
$$

hence choose $\boldsymbol{Q}=\mathbf{0}$.

$$
\begin{aligned}
& \frac{\partial\left(f_{0} / g_{0}\right)}{\partial r} \\
& \quad=-\frac{\left[\kappa\left(1+a_{1} b_{1}\right) \Gamma-\kappa Q-\frac{1}{2}\left(a_{1}+b_{1}\right)^{2}\right]^{2}}{g_{0}^{2}} \\
& \quad<0 ;
\end{aligned}
$$

hence choose $r=r_{0}$.

$\frac{\partial\left(f_{0} / g_{0}\right)}{\partial \Gamma}=\frac{\delta_{1}^{2}-\delta_{2}^{2}}{g_{0}^{2}}$,

where

$\delta_{1}=b_{1} \kappa^{2} \Gamma+\left(r_{0}-\frac{1}{2} \kappa\right)\left(a_{1}+b_{1}\right)$

and

$\delta_{2}=\kappa^{2} \Gamma-\left(r_{0} a_{1}+\frac{1}{2} b_{1}\right)\left(a_{1}+b_{1}\right)$.

Case 1. Setting $\delta_{1}=\delta_{2}$, we obtain

$$
\Gamma=-\frac{a_{1}+b_{1}}{\kappa^{2}}\left(\frac{\kappa}{2}-r_{0} \frac{1+a_{1}}{1-b_{1}}\right)
$$


and

$p=\frac{1+a_{1}}{\kappa^{2}\left(1-b_{1}\right)}\left(\kappa-r_{0} \frac{1+a_{1}}{1-b_{1}}\right)$.

Case 2. Setting $\delta_{1}=-\delta_{2}$, we obtain

$\Gamma=\frac{a_{1}+b_{1}}{\kappa^{2}}\left(\frac{\kappa}{2}-r_{0} \frac{1-a_{1}}{1+b_{1}}\right)$

and

$p=\frac{1-a_{1}}{\kappa^{2}\left(1+b_{1}\right)}\left(\kappa-r_{0} \frac{1-a_{1}}{1+b_{1}}\right)$.

To solve Problem 2 in the feasible region $\Omega_{1}$ is to

$\max f_{1} / g_{1}$

$\begin{array}{ll}\text { subject to } & \Gamma \geq \mathbf{0}, \boldsymbol{Q} \geq \mathbf{0}, r \geq r_{0}, \\ & f_{0}=g_{0}=0, g_{1}>0 .\end{array}$

This problem can be solved similarly and the solution is:

Case 3.

$\Gamma=\frac{1}{2 \kappa} \frac{\left(a_{1}+b_{1}\right)^{2}}{1+a_{1} b_{1}}$,

$Q=\mathbf{0}, \quad r=\frac{\kappa}{2} \frac{1-b_{1}^{2}}{1+a_{1} b_{1}}$,

and

$p=\frac{1}{2 \kappa} \frac{1-a_{1}^{2}}{1+a_{1} b_{1}}$.

Given numeric values of $a_{1}, b_{1}, \kappa$, and $r_{0}$, we can test the feasibility of Cases $1-3$ in $\Omega_{0}$ and $\Omega_{1}$, and compare the maximum values of $p$, of which the largest is the 'best' $p$.

\section{Conclusions}

The max-p problem is formulated for determining a set of 'best' pi-coefficients corresponding to the maximal finite mean-square gain stability range of a feedback system. The original difficult n.s.d. condition on a matrix is transformed into conditions on the coefficients of a series of polynomials. The max-p problem can be more easily solved using the new conditions. Further simplifications to problems involving high-order systems are still under investigation.

\section{References}

[1] D.A. Lawrence and C.R. Johnson, Jr., Recursive parameter identification algorithm stability analysis via pi-sharing, IEEE Trans. Automat. Control 31 (1986) 14.

[2] C.A. Desoer and M. Vidyasagar, Feedback Systems: Input-Output Properties (Academic Press, New York, 1975).

[3] D.A. Lawrence, Adaptive system stability analysis via energy exchange, Ph.D. Dissertation, Cornell University (1985).

[4] D.A. Lawrence and C.R. Johnson, Jr., Output error identification without SPR assumptions, Proc. 23rd IEEE Conf. Decision and Control (Las Vegas, NV, 1984) 977.

[5] K.S. Narendra and R.V. Monopoli, Applications of Adaptive Control (Academic Press, New York, 1980).

[6] K.J. Aström and B. Wittenmark, Adaptive Control (Ad. dison-Wesley, New York, 1989).

[7] B.D.O. Anderson et al., Stability of Adaptive Systems Passivity and Averaging Analysis (MIT Press, Cambridge, MA, 1986).

[8] G.H. Golub and C.F. Van Loan, Matrix Computations (The Johns Hopkins University Press, Baltimore, MD, 1983). 